\title{
Estudo da função auditiva em indivíduos com HIVIAIDS submetidos e não submetidos à terapia antirretroviral
}

\section{Study of hearing functions in individuals with HIVIAIDS submitted and not submitted to antiretroviral therapies}

\author{
Simone Quidicomo', Carla Gentile Matas²
}

\begin{abstract}
RESUMO
Objetivo: Caracterizar as manifestações audiológicas em indivíduos com HIV/AIDS (Vírus da Imunodeficiência Humana/Síndrome da Imunodeficiência Adquirida) submetidos e não submetidos à terapia antirretroviral. Métodos: A avaliação audiológica foi realizada em 28 indivíduos do Grupo Pesquisa I (GPI) com HIV/AIDS, submetidos à terapia antirretroviral; 24 indivíduos do Grupo Pesquisa II (GPII) com HIV/AIDS, não submetidos à terapia antirretroviral e 45 indivíduos saudáveis do Grupo Controle (GC). Os exames audiológicos que compuseram esta pesquisa foram: Audiometria Tonal Convencional, Logoaudiometria e Audiometria em Altas Frequências. Resultados: Houve diferença nos grupos GPI e GPII, para os resultados obtidos tanto na avaliação audiológica convencional, como na avaliação em altas frequências, observando-se limiares auditivos mais elevados quando comparados aos indivíduos do GC, porém sem diferença significativa entre GPI e GPII. Conclusão: Indivíduos com HIV/AIDS apresentam mais alteração na Audiometria Tonal Convencional e na Audiometria em Altas Frequências quando comparados a indivíduos saudáveis (GC) sugerindo comprometimento do sistema auditivo, sendo observada maior ocorrência de perda auditiva na Audiometria em Altas Frequências quando comparada à Audiometria Tonal Convencional nos grupos GPI e GPII. Não houve diferença entre indivíduos com HIV/AIDS submetidos (GPI) e não submetidos (GPII) à terapia antirretroviral para a Audiometria Tonal Convencional e Audiometria em Altas Frequências.
\end{abstract}

Descritores: Síndrome da imunodeficiência adquirida; HIV; Terapia anti-retroviral de alta atividade; Audiometria; Perda auditiva

\begin{abstract}
Purpose: To characterize the audiologic manifestations in individuals with HIV/AIDS (Human Immunodeficiency Virus/Acquired Immunodeficiency Syndrome) submitted and not submitted to antiretroviral therapy. Methods: The audiologic evaluation was carried out in 28 individuals in Research Group I (RGI) with HIV/AIDS submitted to antiretroviral therapy; 24 individuals in Research Group II (RGII) with HIV/AIDS not submitted to antiretroviral therapy, and 45 healthy individuals in the Control Group (CG). The audiologic tests that comprised this study were: Conventional Pure Tone Audiometry, Speech Audiometry and High Frequency Audiometry. Results: There were differences between groups RGI and RGII regarding conventional audiologic evaluation and high frequency audiometry. There were higher thresholds for both Conventional and High Frequency Audiometries when compared with CG subjects, however without significant difference among RGI and RGII. Conclusion: Individuals with HIV/AIDS present more abnormal results in Conventional Pure Tone Audiometry and High Frequency Audiometry than healthy individuals (CG), which indicates impairment in the hearing system. Individuals with HIV/AIDS present more abnormal findings in High Frequency Audiometry when compared to Conventional Pure Tone Audiometry. There was no difference between individuals with HIV/AIDS submitted (RGI) and not submitted (RGII) to antiretroviral therapy regarding Conventional Pure Tone Audiometry and High Frequency Audiometry.
\end{abstract}

Keywords: Acquired immunodeficiency syndrome; HIV; Antiretroviral therapy, Highly active; Audiometry; Hearing loss

Trabalho realizado no Programa de Pós-graduação (Mestrado) em Ciência da Reabilitação, Departamento de Fisioterapia, Fonoaudiologia e Terapia Ocupacional, Faculdade de Medicina, Universidade de São Paulo - USP - São Paulo (SP).

(1) Programa de Pós-graduação (Mestrado) em Ciência da Reabilitação, Departamento de Fisioterapia, Fonoaudiologia e Terapia Ocupacional, Faculdade de Medicina, Universidade de São Paulo - USP - São Paulo (SP), Brasil.

(2) Curso de Fonoaudiologia, Faculdade de Medicina, Universidade de São Paulo - USP - São Paulo (SP), Brasil.

Conflito de interesses: Não

Contribuição dos autores: $S Q$ foi responsável pela coleta de dados e pela pesquisa dos artigos para elaboração do texto; $C G$ orientou o trabalho e colaborou na elaboração do texto.

Endereço para correspondência: Simone Quidicomo. R. Cipotânea, 51, Butantã, São Paulo (SP), Brasil, CEP: 05360-160. E-mail: squidicomo@ uol.com.br Recebido em: 25/3/2012; Aceito em: 8/1/2013 


\section{INTRODUÇÃO}

A Síndrome da Imunodeficiência Adquirida (Acquired Immunodeficiency Syndrome - AIDS - é causada pela ação do vírus da Imunodeficiência Humana (Human Immunodeficiency Virus) - HIV, que fragiliza o sistema imunológico infectando os linfócitos T4, responsáveis pela imunidade celular, e multiplicando-se dentro dele, por meio das células denominadas CD4+. O HIV se instala no organismo destruindo essas células, alterando o funcionamento do sistema imunológico, causando o enfraquecimento das defesas do organismo e favorecendo, desta forma, o início de uma série de doenças denominadas como infecções oportunistas.

Sabe-se que Indivíduos com HIV/AIDS podem apresentar diversas infecções e comprometimentos associados à audição como sintomas característicos da doença.

Estudos na literatura demonstram que a evolução da AIDS pode comprometer o Sistema Nervoso Central (SNC), e alterar o sistema auditivo tanto na sua porção periférica quanto na porção central, justificando assim a investigação da via auditiva como um todo ${ }^{(1-5)}$.

Antigamente, a AIDS era descrita como uma doença de perfil agudo, sendo atualmente considerada pela Organização Mundial da Saúde (OMS) uma doença de perfil crônico, devido aos grandes avanços que foram conseguidos principalmente no que se refere ao seu tratamento, a partir de 1996, utilizando a terapia antirretroviral altamente ativa (highly active antiretroviral therapy - HAART) ${ }^{(6)}$.

Embora a medicação seja necessária e fundamental para muitos pacientes proporcionando melhoras significativas, aumentando a sobrevida desses indivíduos ${ }^{(7)}$, existem relatos na literatura que discutem seus efeitos adversos ${ }^{(8,9)}$.

O comprometimento da orelha interna, decorrente da própria ação do vírus HIV é mencionado em estudos envolvendo pacientes com $\operatorname{AIDS}^{(10-14)}$, assim como a alteração auditiva ocasionada pelo uso de tratamento antirretroviral e medicações ototóxicas ${ }^{(9,15-21)}$, ambos ocasionando perdas auditivas do tipo neurossensorial e, consequentemente, danos irreversíveis à orelha interna.

Além das pesquisas demonstrando que tanto o HIV/AIDS como a medicação utilizada para o tratamento podem afetar o sistema auditivo, outros estudos sugerem que indivíduos com HIV/AIDS seriam mais acometidos por alterações auditivas do que indivíduos sem HIV/AIDS, bem como indivíduos medicados apresentariam mais alterações auditivas quando comparados aos indivíduos não medicados.

Portanto, o objetivo deste estudo foi caracterizar as manifestações audiológicas de indivíduos com HIV/AIDS, submetidos e não submetidos à terapia antirretroviral.

\section{MÉTODOS}

Este estudo foi desenvolvido no Laboratório de Investigação
Fonoaudiólogica em Potenciais Evocados Auditivos do Curso de Fonoaudiologia, do Departamento de Fisioterapia, Fonoaudiologia e Terapia Ocupacional da Faculdade de Medicina da Universidade de São Paulo (FMUSP).

A pesquisa foi realizada na cidade de Santos e envolveu duas unidades municipais distintas: SECRAIDS (Seção - Centro de Referência em AIDS) da Secretaria Municipal de Saúde de Santos, que permitiu a seleção dos indivíduos contaminados pelo HIV; SEVREST (Seção de Vigilância e Referência em Saúde do Trabalhador da Seção) da Secretaria de Saúde da Prefeitura Municipal de Santos, que permitiu a realização dos exames, cedendo seu espaço e equipamentos.

O projeto foi encaminhado para o Comitê de Ética para Análise de Projetos de Pesquisa - CAPPesq da FMUSP, tendo sido aprovado sob protocolo número 389/10.

A casuística foi composta por 97 indivíduos na faixa etária entre 18 e 50 anos de idade, subdivididos em três grupos distintos: Grupo Pesquisa I (GPI) constituído por 28 indivíduos com HIV positivo, submetidos ao tratamento com medicação antirretroviral; Grupo Pesquisa II (GPII) constituído por 24 indivíduos com HIV positivo, não submetidos ao tratamento com medicação antirretroviral; Grupo Controle (GC) constituído por 45 indivíduos saudáveis, sem queixa auditiva e que referiram em anamnese não serem portadores do vírus HIV.

Após a assinatura do Termo de Consentimento Livre e Esclarecido foi realizada a investigação da história clínica do paciente, por meio de anamnese, abordando a presença ou não de queixas auditivas, bem como de doenças que pudessem acometer a audição dos pacientes.

A seguir, foram realizados os seguintes procedimentos para a coleta dos dados: meatoscopia, Audiometria Tonal Convencional (ATC), Limiar de Recepção de Fala (LRF), Índice Percentual de Reconhecimento de Fala (IPRF) e Audiometria em Altas Frequências (AAF). Os equipamentos usados para tal foram: otoscópio Heine ${ }^{\circledR}$; audiômetro Amplaid ${ }^{\circledR}$, modelo A321, com fones TDH 49P para avaliação audiológica convencional e fones HDA 200 para avaliação audiométrica em altas frequências. O LRF foi realizado para confirmar os resultados obtidos na ATC, não sendo analisados estatisticamente.

Os critérios de normalidade adotados para cada procedimento realizado encontram-se descritos a seguir:

- Audiometria Tonal Convencional (ATC): limiares auditivos por via aérea menores ou iguais a $25 \mathrm{~dB}$ NA para as frequências de 250, 500, 1000, 2000, 3000, 4000, 6000 e $8000 \mathrm{~Hz}^{(22)}$. - Audiometria em Altas Frequências (AAF): o critério de normalidade foi estabelecido baseado nos valores médios obtidos no GC deste estudo, considerando um desvio-padrão acima destes valores como corte de normalidade (Quadro 1).

Conforme os critérios estabelecidos, os exames que não apresentaram os resultados anteriormente citados foram considerados alterados, sendo o indivíduo classificado como alterado quando pelo menos uma das orelhas apresentasse alteração. 
Quadro 1. Valores de corte para normalidade na audiometria em altasfrequências (em dB NA), por faixa etária, segundo o grupo controle deste estudo

\begin{tabular}{|lcccccccc|}
\hline Faixa etária & $9 \mathrm{kHz}$ & $10 \mathrm{kHz}$ & $11,2 \mathrm{kHz}$ & $12,5 \mathrm{kHz}$ & $14 \mathrm{kHz}$ & $16 \mathrm{kHz}$ & $18 \mathrm{kHz}$ & $20 \mathrm{kHz}$ \\
\hline $18-29$ anos & 9,6 & 8,8 & 10,8 & 7,4 & 6,7 & 7,0 & 16,3 & 24,1 \\
$30-39$ anos & 16,3 & 15,7 & 15,5 & 16,7 & 19,8 & 34,7 & 37,9 \\
$>40$ anos & 20,4 & 21,0 & 24,5 & 27,1 & 28,8 & 37,4 & 36,3 & 38,3 \\
\hline
\end{tabular}

$\mathrm{Na}$ ATC, os resultados alterados foram classificados como perda auditiva condutiva, neurossensorial ou mista ${ }^{(23)}$, utilizando para tanto a comparação entre os limiares auditivos por via aérea e via óssea (presença ou ausência de gap aéreo-ósseo).

A análise estatística inicialmente foi realizada por meio da avaliação dos dados quantitativos, sendo calculadas as medidas descritivas (média, mediana, desvio-padrão) por orelha, frequência e grupo, sendo utilizado o teste de Friedman na comparação entre orelha direita (OD) e orelha esquerda (OE) por frequência e grupo, bem como o teste de Kruskal-Wallis na comparação entre os três grupos. O nível de significância adotado foi igual a $5 \%$, sendo assinalados com asterisco os valores significativos.

A seguir, a análise qualitativa dos dados foi realizada comparando-se os resultados normais e alterados encontrados em cada grupo e entre os grupos (teste estatístico Qui-quadrado de homogeneidade), sendo descritos os tipos de alteração observados em cada avaliação.

\section{RESULTADOS}

\section{Audiometria Tonal Convencional (ATC)}

Na comparação entre os grupos GC, GPI e GPII para os limiares auditivos nas frequências de 250, 500, 2000, 3000, 6000 e $8000 \mathrm{~Hz}$ (Tabela 1), foi considerada a média entre OD e OE, visto que os resultados obtidos nas duas orelhas não apresentaram diferença entre suas distribuições.

Observou-se nas frequências de 250, 2000, 6000 e 8000 $\mathrm{Hz}$ que os limiares auditivos foram significativamente mais elevados nos grupos com HIV/AIDS quando comparados com o GC, sendo que na comparação dos grupos dois a dois tanto o GPI como o GPII apresentou diferença significativa ao ser comparado com o GC, para as frequências de 250, $6000 \mathrm{e}$ $8000 \mathrm{~Hz}(250 \mathrm{~Hz}: \mathrm{GC}$ x GPI $\mathrm{p}=0,04, \mathrm{GC}$ x GPII $\mathrm{p}=0,04 ; 6000$ Hz: GC x GPI $p=0,002$, GC x GPII $p=0,019 ; 8000 \mathrm{~Hz}$ : GC x GPI $p=0,005$, GC x GPII $p=0,026$ ). Na frequência de 2000 $\mathrm{Hz}$, apenas o GPII apresentou diferença quando comparado ao $\mathrm{GC}(\mathrm{p}=0,008)$.

Uma vez que os limiares auditivos obtidos nas duas orelhas para as frequências de 1000 e $4000 \mathrm{~Hz}$ apresentaram diferença entre suas distribuições, as comparações entre os três grupos foram realizadas separadamente para as OD e OE. Em 1000 $\mathrm{Hz}$ na OD, não houve diferença na comparação entre os grupos $(\mathrm{p}=0,208)$, sendo que esta diferença esteve presente na $\mathrm{OE}(\mathrm{p}=0,003)$, estando os limiares auditivos menores no GC quando comparados aos grupos GPI e GPII ( $\mathrm{p}=0,021 \mathrm{e} \mathrm{p}=0,002$ respectivamente), porém sem diferença entre os grupos com

Tabela 1. Estatísticas descritivas para a variável ATC por frequência e grupo

\begin{tabular}{|c|c|c|c|c|c|}
\hline Frequência & Grupo & Média & Mediana & Desvio-padrão & $\begin{array}{c}\text { Valor de } p \\
\text { (entre grupos) }\end{array}$ \\
\hline \multirow{3}{*}{$250 \mathrm{~Hz}$} & $\mathrm{GC}$ & 11,78 & 12,50 & 4,15 & \multirow{3}{*}{$0,044^{*}$} \\
\hline & GPI & 14,46 & 15,00 & 5,37 & \\
\hline & GPII & 14,17 & 13,75 & 4,40 & \\
\hline \multirow[b]{2}{*}{$500 \mathrm{~Hz}$} & GC & 10,22 & 10,0 & 3,69 & \multirow[b]{2}{*}{0,119} \\
\hline & GPI & 12,05 & 12,5 & 4,81 & \\
\hline \multirow[t]{2}{*}{$2000 \mathrm{~Hz}$} & GPI & 8,66 & 8,75 & 6,99 & \multirow[t]{2}{*}{$0,032^{*}$} \\
\hline & GPII & 9,17 & 8,75 & 4,34 & \\
\hline \multirow{3}{*}{$3000 \mathrm{~Hz}$} & GC & 8,44 & 7,50 & 5,44 & \multirow{3}{*}{0,067} \\
\hline & GPI & 10,27 & 8,75 & 6,71 & \\
\hline & GPII & 11,35 & 11,25 & 5,05 & \\
\hline \multirow{3}{*}{$8000 \mathrm{~Hz}$} & GC & 8,10 & 7,50 & 5,36 & \multirow{3}{*}{$0,008^{*}$} \\
\hline & GPI & 13,75 & 11,25 & 9,61 & \\
\hline & GPII & 13,75 & 10,00 & 10,91 & \\
\hline
\end{tabular}

*Valores significativos $(p<0,005)-$ Teste Kruskal Wallis

Legenda: ATC = Audiometria Tonal Convencional; GC = Grupo Controle; GPI = Grupo Pesquisa I; GPII = Grupo Pesquisa II 
Tabela 2. Distribuição da ocorrência de resultados normais e alterados na ATC nos grupos GC, GPI e GPII

\begin{tabular}{|c|c|c|c|c|c|c|c|c|}
\hline \multirow{3}{*}{$\begin{array}{l}\text { Audiometria tonal } \\
\text { convencional }\end{array}$} & \multicolumn{8}{|c|}{ Grupo } \\
\hline & \multicolumn{2}{|c|}{$\mathrm{GC}$} & \multicolumn{2}{|c|}{ GPI } & \multicolumn{2}{|c|}{ GPII } & \multicolumn{2}{|c|}{ Total } \\
\hline & $\mathrm{n}$ & $\%$ & $\mathrm{n}$ & $\%$ & $\mathrm{n}$ & $\%$ & $\mathrm{n}$ & $\%$ \\
\hline Normal & 45 & 100 & 23 & 82,14 & 19 & 79,17 & 87 & 89,69 \\
\hline Alterado & 0 & 0 & 5 & 17,86 & 5 & 20,83 & 10 & 10,31 \\
\hline Total & 45 & 100 & 28 & 100 & 24 & 100 & 97 & 100 \\
\hline
\end{tabular}

Teste Qui-quadrado de homogeneidade $(p=0,008)$

Legenda: ATC = Audiometria Tonal Convencional; GC = Grupo Controle; GPI = Grupo Pesquisa I; GPII = Grupo Pesquisa II

HIV/AIDS (p=0,275). Em $4000 \mathrm{~Hz}$ houve diferença na comparação entre os grupos, tanto na OD como na $\mathrm{OE}(\mathrm{p}=0,003 \mathrm{e}$ $\mathrm{p}=0,031$ respectivamente), evidenciando-se limiares auditivos menores para o GC quando comparado ao GPI (OD: $\mathrm{p}=0,001$; OE: $\mathrm{p}=0,007)$.

Na distribuição da ocorrência de resultados normais e alterados na ATC, verificou-se que indivíduos com HIV/AIDS (GPI e GPII) apresentaram mais alteração quando comparados a indivíduos do GC (Tabela 2).

Ao comparar os grupos dois a dois, não foi possível observar diferença entre os resultados normais e alterados obtidos nos grupos com HIV/AIDS submetidos e não submetidos à terapia antirretroviral $(\mathrm{p}>0,999)$, porém, os resultados foram significativos quando comparados ao GC (GPI: $p=0,007$ e GPII: $p=0,004$ ).

Com relação aos tipos de alterações encontradas na ATC, observou-se maior ocorrência de perda auditiva do tipo neurossensorial. Para o GPI, três indivíduos apresentaram perda auditiva neurossensorial unilateral e dois, perda bilateral. Para o GPII, dois indivíduos apresentaram perda auditiva neurossensorial unilateral, dois indivíduos apresentaram alteração neurossensorial bilateral e um indivíduo apresentou perda auditiva condutiva unilateral.

\section{Audiometria em Altas Frequências (AAF)}

Na comparação entre os grupos GC, GPI e GPII para os limiares auditivos (nas frequências de 9, 10, 11,2, 12,5, 14, 16 e $18 \mathrm{kHz}$ ) obtidos na AAF (Tabela 3), foi considerada a média entre $\mathrm{OD}$ e $\mathrm{OE}$, visto que os resultados obtidos nas duas orelhas não apresentaram diferença.

Observou-se nas frequências de 10,11,2, 12,5, 14 e 18 $\mathrm{kHz}$ que os limiares auditivos foram significativamente mais elevados nos grupos com HIV/AIDS quando comparados com o GC, sendo que na comparação dos grupos dois a dois apenas

Tabela 3. Estatísticas descritivas para a variável AAF por frequência e grupo

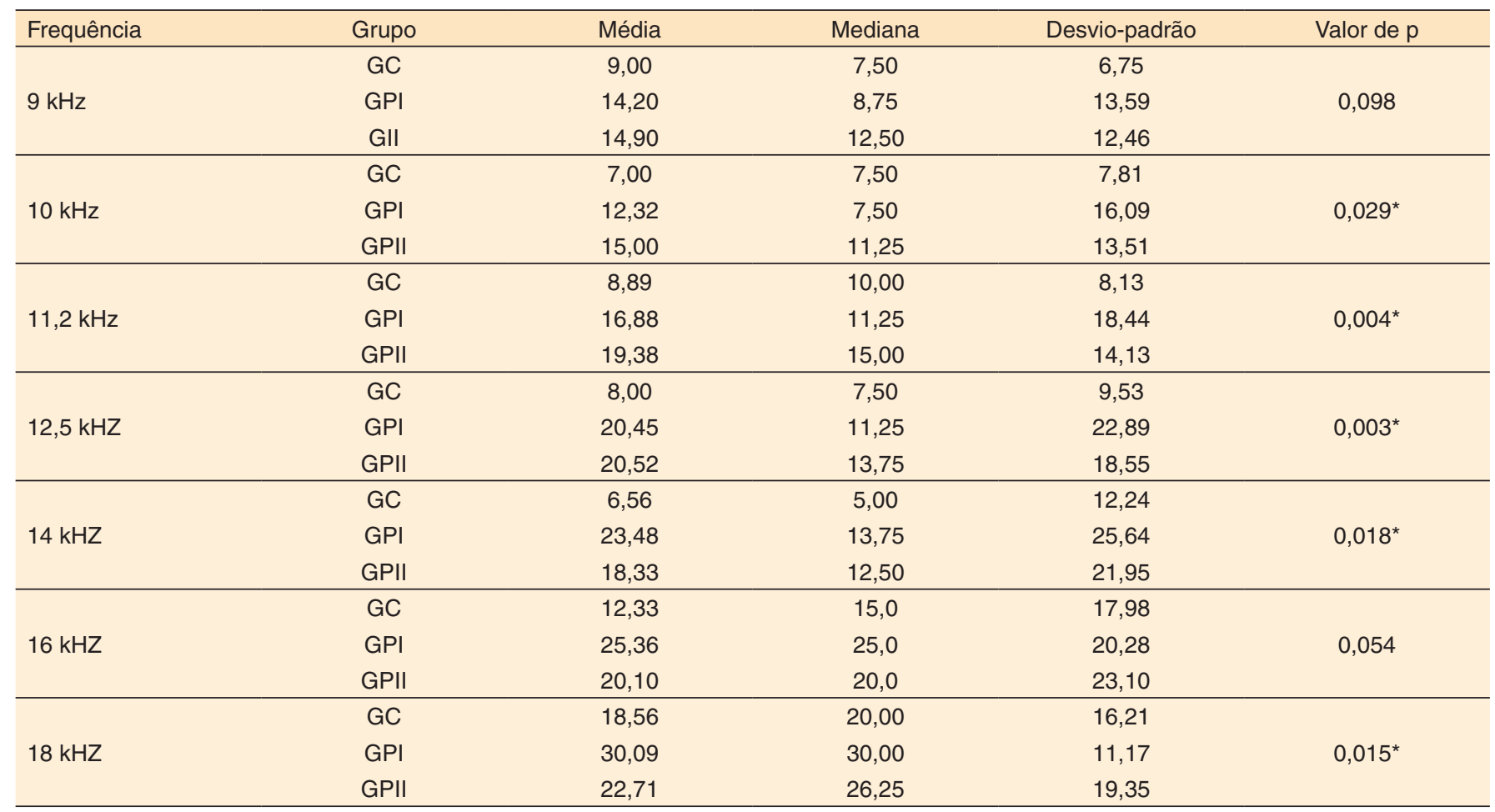

*Valores significativos $(p<0,005)-$ Teste Kruskal Wallis

Legenda: AAF= Audiometria em Altas Frequências; GC = Grupo Controle; GPI = Grupo Pesquisa I; GPII = Grupo Pesquisa II 
Tabela 4. Distribuição da ocorrência de resultados normais e alterados na AAF nos grupos GC, GPI e GPII

\begin{tabular}{|c|c|c|c|c|c|c|c|c|}
\hline \multirow{3}{*}{$\begin{array}{l}\text { Audiometria em } \\
\text { Altas Frequências }\end{array}$} & \multicolumn{8}{|c|}{ Grupo } \\
\hline & \multicolumn{2}{|c|}{ GC } & \multicolumn{2}{|c|}{ GPI } & \multicolumn{2}{|c|}{ GPII } & \multicolumn{2}{|c|}{ Total } \\
\hline & $\mathrm{n}$ & $\%$ & $n$ & $\%$ & $\mathrm{n}$ & $\%$ & $\mathrm{n}$ & $\%$ \\
\hline Normal & 45 & 100 & 10 & 35,71 & 4 & 16,67 & 59 & 60,82 \\
\hline Alterado & 0 & 0 & 18 & 64,29 & 20 & 83,33 & 38 & 39,18 \\
\hline Total & 45 & 100 & 28 & 100 & 24 & 100 & 97 & 100 \\
\hline
\end{tabular}

Teste Qui-quadrado de homogeneidade $(p<0,001)$

Legenda: AAF= Audiometria em Altas Frequências; GC = Grupo Controle; GPI = Grupo Pesquisa I; GPII = Grupo Pesquisa II

o GPI apresentou diferença ao ser comparado com o GC em 14 e $18 \mathrm{kHz}(\mathrm{p}=0,008$ e $\mathrm{p}=0,003$, respectivamente) e, em 10 e $11,2 \mathrm{kHz}$, apenas o GPII apresentou esta diferença quando comparado ao $\mathrm{GC}$ ( $\mathrm{p}=0,007$ e $\mathrm{p}=0,001$, respectivamente).

Uma vez que os limiares auditivos obtidos nas duas orelhas para a frequência de $20 \mathrm{kHz}$ da AAF apresentaram diferença entre suas distribuições, as comparações entre os três grupos foram realizadas separadamente para a OD e OE. Houve diferença na comparação entre os grupos, tanto na OD como na $\mathrm{OE}(\mathrm{p}=0,049$ e $\mathrm{p}=0,05$, respectivamente), evidenciando-se limiares auditivos menores para o GC quando comparado ao GPI (OD: $p=0,016$; OE: $\mathrm{p}=0,018$ ).

Na distribuição da ocorrência de resultados normais e alterados obtidos na AAF verificou-se, também, que indivíduos com HIV/AIDS apresentaram mais alteração quando comparados aos indivíduos do GC (Tabela 4).

Na comparação entre os grupos, não houve diferença entre os resultados normais e alterados obtidos nos indivíduos com HIV/AIDS submetidos e não submetidos à terapia antirretroviral $(\mathrm{p}=0,209)$. A proporção de indivíduos com resultado normal foi maior no $\mathrm{GC}(\mathrm{p}<0,001)$, enquanto que nos grupos GPI e GPII houve uma maior proporção de resultados alterados $(\mathrm{p}=0,001)$.

Os indivíduos com HIV/AIDS também foram avaliados em relação à distribuição de ocorrência de resultados normais e alterados na ATC e na AAF (Tabela 5), observando-se diferença na comparação entre os testes $(\mathrm{p}<0,001)$, sendo que a AAF apresentou maior ocorrência de resultados alterados $(73,08 \%)$ quando comparada à ATC (19,23\%).

Tabela 5. Comparação dos resultados obtidos na ATC e na AAF, nos grupos GPI e GPII

\begin{tabular}{lcccccc}
\hline \multirow{2}{*}{ Resultado } & \multicolumn{2}{c}{ ATC } & \multicolumn{2}{c}{ AAF } & \multicolumn{2}{c}{ Total } \\
\cline { 2 - 7 } & $\mathrm{n}$ & $\%$ & $\mathrm{n}$ & $\%$ & $\mathrm{n}$ & $\%$ \\
\hline Normal & 42 & 80,77 & 14 & 26,92 & 56 & 53,85 \\
Alterado & 10 & 19,23 & 38 & 73,08 & 48 & 46,15 \\
\hline Total & 52 & 100 & 52 & 100 & 104 & 100 \\
\hline
\end{tabular}

Teste Qui-quadrado de homogeneidade $(p<0,001)$

Obs.: Avaliação dos grupos juntos, excluindo o Grupo Controle, que era normal na ATC e na AAF

Legenda: $A T C=$ Audiometria Tonal Convencional; AAF= Audiometria em Altas Frequências; GPI = Grupo Pesquisa I; GPII = Grupo Pesquisa II

\section{DISCUSSÃO}

As manifestações do HIV/AIDS no sistema auditivo são relatadas em estudos que demonstram o acometimento tanto de sua porção periférica como central. É importante ressaltar que as alterações existentes podem ocorrer pela ação do próprio vírus no nosso organismo, devido à imunossupressão e à presença de infecções oportunistas, bem como pela ação dos medicamentos antirretrovirais e medicamentos com potencial ototóxico $^{(5,9-21,24)}$.

A literatura especializada mostra resultados bem diversificados quanto à ocorrência de perda auditiva na população com HIV/AIDS. Estudos referem que aproximadamente 21 a $49 \%$ dos indivíduos com HIV/AIDS podem apresentar perda auditiva $^{(1,9-12,21)}$.

Os achados obtidos no presente estudo mostram que, para ATC, os resultados foram menos expressivos quando comparados aos estudos descritos anteriormente, mas a presença do vírus HIV parece ter interferido nos resultados, observando-se maior ocorrência de resultados alterados nos grupos GPI e GPII, quando comparados ao GC (Tabela 2).

Verificou-se a ocorrência de perda auditiva em $17,86 \%$ no GPI e em 20,83\% no GPII. Para o GPI, dos cinco indivíduos com alteração na ATC, a perda auditiva neurossensorial esteve presente em $100 \%$ dos casos $(60 \%$ com acometimento unilateral e $40 \%$ bilateral). No GPII, dos cinco indivíduos com resultados alterados na ATC, $80 \%$ apresentaram perda auditiva neurossensorial (50\% unilateral e $50 \%$ bilateral) e $20 \%$ perda auditiva condutiva (unilateral).

Diversos trabalhos na literatura consultada referem que o tipo de perda auditiva mais frequentemente observado em adultos com HIV/AIDS é o neurossensorial ${ }^{(5,14,21,24)}$, seguido da perda auditiva condutiva ${ }^{(21)}$, e que o comprometimento é geralmente bilateral e progressivo ${ }^{(2,9,15)}$.

Em relação ao comprometimento de orelha média e, consequentemente, a presença de perda auditiva condutiva ou mista, alguns estudos relatam que esta alteração seria decorrente da imunossupressão, que favorece a presença de infecções oportunistas $^{(25)}$.

Nota-se, no presente estudo, uma maior ocorrência de perda auditiva neurossensorial quando comparada a perda auditiva condutiva na ATC, demonstrando, desta forma, o 
comprometimento da orelha interna nesta população. Os resultados encontrados corroboram um estudo em que o autor, com o objetivo de caracterizar os resultados da avaliação audiológica de crianças e adultos com HIV/AIDS, observou maior ocorrência de perda auditiva do tipo condutiva em crianças $(92,9 \%$ dos casos), enquanto que na população adulta evidenciou-se maior ocorrência de perda auditiva do tipo neurossensorial $(44,4 \% \text { dos casos) })^{(26)}$.

Ao comparar indivíduos com HIV/AIDS submetidos e não submetidos à terapia antirretroviral, os dados obtidos no presente estudo demonstram que não houve diferença quanto à ocorrência de perda auditiva entre estes dois grupos, corroborando outros estudos específicos na área ${ }^{(14,19,20)}$, e discordando de outros estudos que correlacionam o uso de medicação antirretroviral com uma maior ocorrência de alterações auditivas $^{(5,9,15-18,21)}$.

Considerando os dados encontrados na presente pesquisa pode-se observar que a perda auditiva neurossensorial parece estar associada à presença do vírus HIV e seus efeitos adversos no organismo do indivíduo infectado, concordando com outros estudos relatados na literatura ${ }^{(10-12,14,24,25)}$.

Os resultados do presente estudo divergem dos obtidos em outro estudo com pacientes submetidos e não submetidos a tratamento antirretroviral, no qual observou-se maior ocorrência de perda auditiva condutiva no grupo submetido ao tratamento $(30,8 \%)$ quando comparado ao não submetido $(20 \%)$, sendo este achado justificado pelo fato do grupo que faz uso de medicação ser composto por indivíduos com maior tempo de infecção e imunossupressão mais acentuada ${ }^{(26)}$.

A AAF é um exame que tem mostrado grande potencialidade para a detecção precoce de alteração auditiva, pois a região basal da cóclea, onde se encontram as células ciliadas, são as primeiras a serem acometidas nas alterações auditivas causadas por fatores endógenos e exógenos, permitindo o conhecimento destas alterações antecipadamente à $\mathrm{ATC}^{(27,28)}$.

$\mathrm{O}$ monitoramento auditivo de indivíduos submetidos à medicação ototóxicas por meio da AAF propiciou evidências precoces sobre o limite da dose de medicamentos que causam perda auditiva, permitindo a prevenção ou redução da severidade da ototoxicidade.

$\mathrm{Na}$ amostra deste estudo, os resultados encontrados na AAF em indivíduos com HIV/AIDS foram mais expressivos quando comparados aos obtidos na ATC (Tabela 5). Na comparação dos grupos GPI e GPII com o GC (Tabela 4), observou-se diferença significativa $(\mathrm{p}<0,001)$, sendo que o GPI apresentou 64,29\% de resultados alterados e o GPII 83,33\% na AAF.

Estes achados corroboram diversos estudos na literatura que evidenciaram a presença de alterações significativas na $\mathrm{AAF}^{(9,21,26)}$. Em um estudo que avaliou 25 indivíduos com HIV, encontrou-se $88 \%$ dos casos com resultados alterados na $\mathrm{AAF}^{(21)}$. Pesquisando a audição de 56 indivíduos com HIV/ AIDS, estudiosos encontraram 90,2\% de alteração na AAF, com prevalência de $84,2 \%$ no grupo não submetido à medicação e de
95,5\% no grupo submetido à terapia antirretroviral; enfatizando que a AAF pode ser considerada um dos procedimentos mais sensíveis para a identificação precoce da perda auditiva nesta população ${ }^{(9)}$, devendo ser utilizada também no monitoramento auditivo,

Os valores expressivos de alteração auditiva encontrados na AAF na presente pesquisa corroboram os estudos citados anteriormente e enfatizam a importância da realização da $\mathrm{AAF}$ como exame de rotina clínica em indivíduos com HIV/AIDS, demonstrando ser este um procedimento importante e eficiente para o diagnóstico precoce de alterações auditivas, uma vez que estas poderiam não ser observadas precocemente na ATC.

No presente estudo, não foi possível correlacionar os efeitos da terapia antirretroviral nas avaliações audiológicas comportamentais realizadas. Embora alguns autores não tenham conseguido excluir ou correlacionar o efeito da medicação às alterações auditivas ${ }^{(19,20)}$, outros estudos demonstraram diferenças significativas entre indivíduos com HIV/AIDS submetidos e não submetidos à terapia antirretroviral que merecem ser consideradas, principalmente em relação à $\mathrm{AAF}^{(9,21,26)}$. Cabe ressaltar que talvez esta diferença entre os dois grupos (submetidos e não submetidos à terapia antirretroviral) não tenha sido evidenciada devido ao fato do vírus HIV acometer o sistema auditivo periférico precocemente, ocasionando infecções oportunistas como descrito em estudos na área ${ }^{(10-12,14,25)}$, fazendo com que estas alterações auditivas sejam constatadas também em indivíduos que ainda não façam uso da medicação.

Com o avanço das pesquisas voltadas ao estudo do sistema auditivo de indivíduos com HIV/AIDS e da AAF, a ciência permitirá ao longo do tempo que os pacientes possam ser monitorados, evitando maiores danos auditivos e, quem sabe ainda, esse monitoramento possa ser usado para ajuste da medicação ou, até mesmo, sua substituição. Ainda há muito que se aprender com esta doença, lembrando que as alterações auditivas compõem uma pequena parte de uma série de alterações decorrentes da Síndrome da Imunodeficiência Adquirida.

\section{CONCLUSÃO}

Frente aos resultados obtidos pode-se concluir que indivíduos com HIV/AIDS apresentam mais alterações audiológicas quando comparados a indivíduos saudáveis, sugerindo comprometimento do sistema auditivo. A AAF apresentou maior ocorrência de perda auditiva quando comparada a ATC. Não foi possível observar, neste estudo, diferenças em relação aos resultados obtidos na ATC e na AAF entre indivíduos com HIV/ AIDS submetidos e não submetidos à terapia antirretroviral.

\section{REFERÊNCIAS}

1. Madriz JJ, Herrera G. Human immunodeficiency virus and acquired immune deficiency syndrome AIDS - related hearing disorders. J Am Acad Audiol. 1995Sep;6(5):358-64. 
2. Bankaitis AE, Christensen LA, Murphy G, Morehouse CR. HIV/ AIDS and auditory evoked potentials. Semin Hear. 1998;19(2):17793.

3. Matkin ND, Diefendorf AO, Erenberg A. Children: HIV/AIDS and hearing loss. SeminHear.1998;19(2):143-53.

4. Matas CG, Silva SM, Marcon BA, Gonçalves IC. Manifestações eletrofisiológicas em adultos com HIV/AIDS submetidos e nãosubmetidos à terapia anti-retroviral. Pro Fono. 2010;22(2):107-12.

5. Matas CG. Caracterização das manifestações audiológicas e eletrofisiológicas em adultos HIV positivos [tese livre docência]. São Paulo: Faculdade de Medicina, Universidade de São Paulo; 2010b.

6. Alencar TMD, Nemes MIB, Velloso MA. Transformação da "aids aguda" para a "aids crônica": percepção corporal e intervenções cirúrgicas entre pessoas vivendo com HIV e AIDS. Ciênc Saúde Colet. 2008;13(6):1841-9

7. Christo PP. Alterações Cognitivas na infecção pelo HIV e AIDS. Rev Assoc Med Bras. 2010;56(2):242-7

8. Geocze L, Mucci S, De Marco MA, Nogueira-Martins LA, Citero VA. Qualidade de vida e adesão ao tratamento anti-retoviral de pacientes portadores de HIV. Rev Saúde Pública. 2010;44(4):743-9.

9. Matas CG, Marcon BA, Silva SM, Gonçalves IC. Avaliação auditiva na Síndrome da Imunodeficiência Adquirida. Rev Soc Bras Fonoaudiol. 2010;15(2):174-8.

10. Domenech J, Fuste J, Traserra J. Equilibrium and auditory disorders in patients affected by HIV-1. Rev Neurol. 1996Dec;24(136):1623-6.

11. Mata Castro N, Yebra Bango M, Tutor de Ureta P, Villarreal Garcia-Lomas M, Garcia Lopez F. Hearing loss and human immunodeficiency virus infection. Study of 30 patients. Rev Clin Esp. 2000May;200(5):271-4.

12. Khoza K, Ross E. Auditory function in a group of adults infected with HIV/AIDS in Gauteng, South Africa. S Afr J Commun Disord. 2002;49:17-27.

13. Neto CDP, Weber R, Araújo-Filho BC, Miziara ID. Rinossinusites em crianças infectadas pelo HIV sob terapia anti-retroviral. Rev Bras Otorrinolarigol. 2009;75(1):70-5.

14. Vieira ABC, Mancini P, Gonçalves DU. Doenças infecciosas e perda auditiva. Rev Med Minas Gerais. 2010;20(1):102-6.

15. Marra CM, Wechkin HA, Longstreth WT Jr, Rees TS, Syapin CL, Gates GA. Hearing loss and anti-retroviral therapy in patients infected with HIV-1. Arch Neurol. 1997;54(4):407-10.
16. Christensen LA, Morehouse CR, Powell TW, Alchediak T, Silio M. Antiviral therapy in a child with pediatric human immunodeficiency virus (HIV): case study of audiologic findings. J Am Acad Audiol. 1998Aug;9(4):292-8.

17. Simdom J, Watters D, Bartlett S, Connick E. Ototoxicity associated with use of nucleoside analog reverse transcriptase inhibitors: a report of 3 possible cases and review of the literature. Clin Infect Dis. 2001Jun;32(11):1623-7.

18. Williams B. Ototoxicity may be associated with protease inhibitor therapy. Clin Infect Dis. 2001Dec;33(12):2100-01.

19. Campanini A, Marani M, Mastroianni A, Cancellieri C, Vicini C. Human immunodeficiency virus infection: personal experience in changes in head and neck manifestations due to recent anti-retroviral therapies. Acta Otorhinolaryngol Ital. 2005Feb;25(1):30-5.

20. Vieira ABC, Greco DB, Teófilo MMM, Gonçalves DU. Manifestações otoneurológicas associadas à terapia anti-retroviral. Rev Soc Bras Med Trop. 2008Jan-Fev;41(1):65-9.

21. Juan KR. Avaliação audiológica, eletroacústica e eletrofisiológica da audição em adultos com HIV/AIDS [dissertação]. São Paulo: Faculdade de Medicina, Universidade de São Paulo, 2009.

22. Lloyd LL, Kaplan, H. Audiometric interpretation: a manual o basic audiometry. Baltimore: University Park Press; 1978. p. 16-7, 94.

23. Silman S, Silverman CA. Basic audiologic testing. In: Silman S, Silverman CA. Auditory diagnosis: principles and applications. San Diego: Singular Publishing Group, 1997. p.44-52.

24. Sanjar FA, Queiroz BEUP, Miziara ID. Otolarigologic manifestations in HIV disease: clinical aspects and treatament. Braz J Otorhinolaryngol. 2011;77(3):391-400.

25. Prasad HKC, Bhojwani KM, Shenoy V, Prasad SC. HIV manifestation in otolaryngology. Am J Otolaryngol. 2006MayJun;27(3):179-85.

26. Matas CG, Santos Filha VAV, Juan KR, Pinto FR, Gonçalves IC. Manifestações audiológicas em crianças e adultos com AIDS. Pro Fono. 2010Jul-Set;22(3):269-74.

27. Klagenberg KF, Oliva FC, Gonçalves CGO, Lacerda ABM, Garofani VG, Zeigelboim BS. Audiometria de altas frequências no diagnóstico complementar em audiologia: uma revisão da literatura nacional. Rev Soc Bras Fonoaudiol. 2011;16(1):109-14.

28. Jacob LCB, Aguiar FP, Tomiasi AA, Tschoeke SN, Bitencourt RF. Monitoramento auditivo na ototoxidade. Rev Bras Otorrinolaringol. 2006Dec;72(6):836-44. 